\title{
Beam injection with a pulsed sextupole magnet in an electron storage ring
}

\author{
Hiroyuki Takaki and Norio Nakamura \\ Institute for Solid State Physics, The University of Tokyo, 5-1-5 Kashiwanoha, Kashiwa, Chiba 277-8581, Japan
}

Yukinori Kobayashi, Kentaro Harada, Tsukasa Miyajima, Akira Ueda, Shinya Nagahashi, Miho Shimada, Takashi Obina, and Tohru Honda

Photon Factory, High Energy Accelerator Research Organization, 1-1 Oho, Tsukuba, Ibaraki 305-0801, Japan

(Received 8 February 2009; published 24 February 2010)

\begin{abstract}
We have developed a new beam injection system with a single pulsed sextupole magnet (PSM) at the photon factory storage ring (PF ring) in the High Energy Accelerator Research Organization. We demonstrated beam injection with this system and succeeded in storing a beam current of $450 \mathrm{~mA}$, which is the normal operating beam current of the PF ring. Top-up injection was also achieved. Coherent dipole oscillation of the stored beam and fluctuation of photon intensity observed at synchrotron radiation beam lines during the PSM injection became very small compared with a pulsed bump injection system that is normally used in the beam injection at the PF ring. This experiment is the first demonstration of beam injection using the PSM in an electron storage ring.
\end{abstract}

DOI: 10.1103/PhysRevSTAB.13.020705

PACS numbers: 29.27.Ac, 07.55.Db, 29.20.db, 41.60.Ap

\section{INTRODUCTION}

With the spread of "top-up" injection for synchrotron radiation (SR) sources, it has become very important to suppress stored beam oscillation during beam injection [1]. In top-up injection, a stored beam current in an electron storage ring is kept at a constant value by continuous beam injection to conduct high-flux SR experiments with constant SR heat load on beam line optics and to eliminate current dependent systematic errors in the SR experiments. In conventional beam injection for electron storage rings, a pulsed local bump produced by several kicker magnets is employed to reduce the amplitude of coherent dipole oscillation in the injected beam. Since the kicker magnet is an established technology, this method has been adopted in most electron storage rings. However, it is difficult to provide the complete closed bump because of the magnetic field errors, timing jitters, and nonlinear effects such as from sextupole magnets inside the bump [2]. The unclosed bump generates the coherent dipole oscillation of the stored beam, which degrades the quality of the photon beam for the SR experiments in top-up injection.

To solve this problem, we have developed a new beam injection method using a pulsed sextupole magnet (PSM) without the pulsed bump and examined the performance at the photon factory storage ring (PF ring) [3-5]. In this method, the injected beam is captured into the ring acceptance by a kick of the PSM while the stored beam passes through the center of the PSM where the magnetic field is almost zero. Thus, the method allows us to realize a highquality photon beam for SR users without large coherent oscillation of the stored beam even in top-up injection. In this paper, we describe the first demonstration of beam injection using a PSM carried out in an electron storage ring.

\section{PRINCIPLE}

\section{A. PSM injection method}

First, we consider the behavior of the injected beam in PSM injection. The injected beam circulates with a large amplitude of coherent dipole oscillation in a storage ring just after the injection. The initial amplitude is determined by the distance from a central orbit at the injection point. To capture the injected beam within the storage ring acceptance, we have to reduce the amplitude using the pulsed magnets. Figures 1(a) and 1(b) show schematic views of the conventional injection and the PSM injection. In the

(a) Pulsed bump injeciton

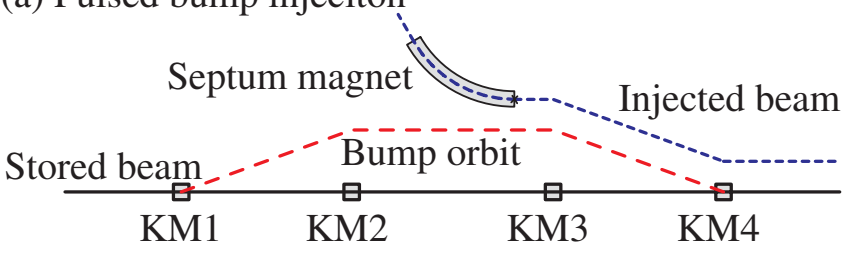

(b) PSM injeciton

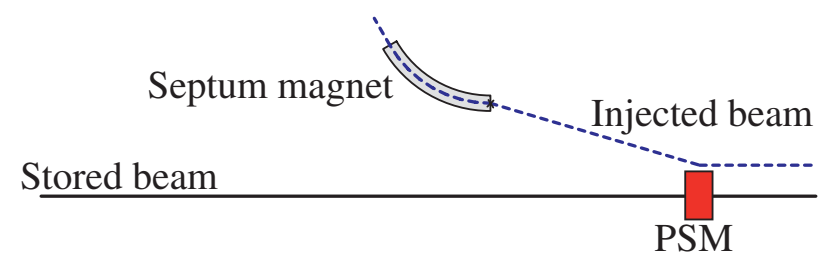

FIG. 1. (Color) A schematic view of beam injection. (a) Conventional pulsed bump injection. The bump orbit (red) is employed by four pulsed dipole kicker magnets (KM1-KM4). Trajectories of the injected beam (blue) and the stored beam (black) are shown. (b) PSM injection. 
conventional injection, a pulsed local bump is produced by kicker magnets (KM1-KM4) for the stored beam, and the coherent dipole oscillation of the injected beam is decreased by the downstream kicker magnets (KM3 and KM4). The bumped orbit ordinarily disappears after one turn of the beam around the ring to prevent the injected beam from hitting the septum magnet wall. On the other hand, in the PSM injection, the oscillation amplitude is reduced by only one pulsed magnet as shown in Fig. 1.

The trajectory of the injected beam in PSM injection is illustrated schematically using a normalized phase space in Fig. 2. The normalized coordinates are defined by

$$
X=\frac{x}{\sqrt{\beta}}, \quad P=\frac{\alpha x+\beta x^{\prime}}{\sqrt{\beta}},
$$

where $x$ and $x^{\prime}$ are the horizontal position and the angle of the beam; $\alpha$ and $\beta$ are the Twiss parameters in the horizontal direction. The trajectory starts from the injection point $\left(X_{0}, P_{0}\right)$ and moves along the outer circle in the clockwise direction (the red line of the figure). The radius of the outer circle in the phase space is the square root of the Courant-Snyder invariant represented by $A_{\text {inj }}$, which we call "injection amplitude,"

$$
A_{\mathrm{inj}}^{2}=X_{0}^{2}+P_{0}^{2}=\gamma_{0} x_{0}^{2}+2 \alpha_{0} x_{0}^{\prime} x_{0}+\beta_{0} x_{0}^{\prime 2},
$$

where $\gamma_{0}=\left(1+\alpha_{0}^{2}\right) / \beta_{0}$, and the suffix " 0 " represents the injection point. Because the radiation damping time is tens of thousands of times larger than the revolution period

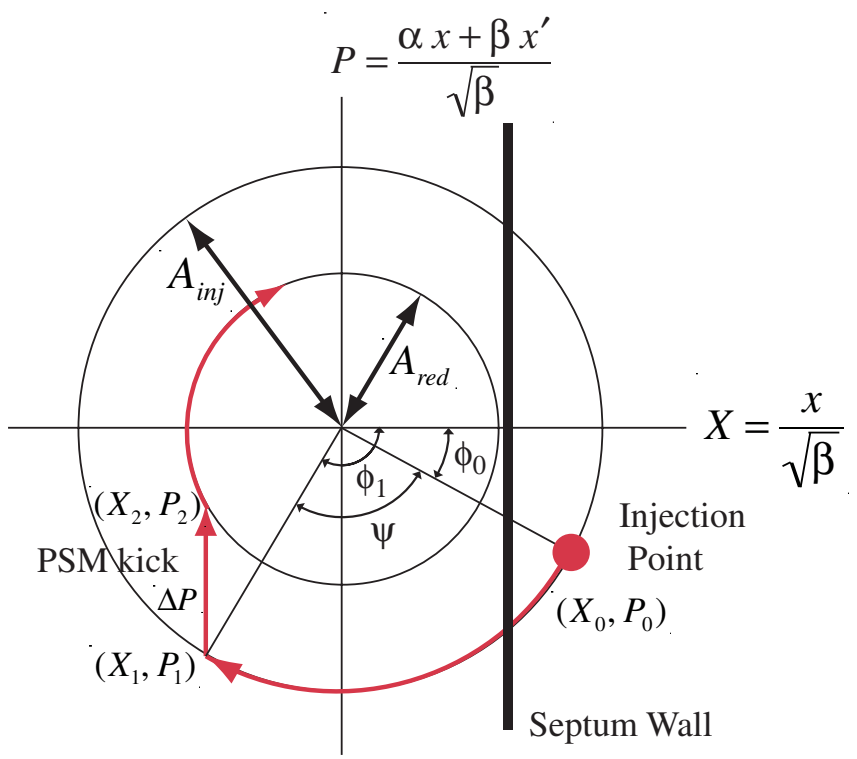

FIG. 2. (Color) A trajectory of an injected beam in PSM injection (red) on a normalized phase space. $\left(X_{0}, P_{0}\right),\left(X_{1}, P_{1}\right)$, and $\left(X_{2}, P_{2}\right)$ are the positions of the injected beam at the injection point, just before and after the kick at the PSM location. The radius of the outer circle, $A_{\mathrm{inj}}$, is the square root of the CourantSnyder invariant for the injected beam before the PSM kick, and that of the inner circle, $A_{\text {red }}$, is one after the PSM kick. of the ring, $A_{\text {inj }}$ is considered constant in the first several turns of the injected beam around the ring.

The beam position at the injection point is also expressed using the "phase angle" $\phi_{0}$ which is the angle of the injection point to the $X$ axis in the clockwise direction as shown in Fig. 2:

$$
X_{0}=A_{\text {inj }} \cos \phi_{0}, \quad P_{0}=-A_{\text {inj }} \sin \phi_{0} .
$$

The beam position at the PSM position, $\left(X_{1}, P_{1}\right)$, is expressed with the phase angle $\phi_{1}$ in the same manner,

$$
X_{1}=A_{\text {inj }} \cos \phi_{1}, \quad P_{1}=-A_{\text {inj }} \sin \phi_{1} .
$$

The relation between $\phi_{0}$ and $\phi_{1}$ is represented by the phase advance $\psi$ from the injection point to the PSM,

$$
\psi=\int_{X_{0}}^{X_{1}} \frac{1}{\beta(s)} d s=\phi_{1}-\phi_{0} .
$$

If we do nothing to the injected beam, it revolves in the ring and finally hits the wall of the septum magnet in several turns depending on its fractional horizontal operating tune. To avoid beam loss, the injection amplitude should be reduced by the PSM kick to the inner circle, which is defined as "reduced injection amplitude,"

$$
A_{\text {red }}^{2}=X_{2}^{2}+P_{2}^{2}
$$

where $\left(X_{2}, P_{2}\right)$ represents the beam position just after the PSM kick. The kick angle of the PSM for the injected beam is expressed by

$$
\theta=-\frac{1}{2} K_{2} x_{1}^{2}
$$

where $K_{2}\left(=B^{\prime \prime} \ell / B \rho\right)$ denotes the sextupole field strength. The positive value of $\theta$ represents the outside direction of the ring, and a focusing sextupole magnet has positive $K_{2}$. Using the thin lens approximation, the injected beam position $\left(X_{2}, P_{2}\right)$ is expressed by the PSM kick of $\theta$,

$$
\begin{aligned}
& X_{2}=\frac{x_{1}}{\sqrt{\beta_{1}}}=X_{1}, \\
& P_{2}=\frac{\alpha_{1} x_{1}+\beta_{1}\left(x_{1}^{\prime}+\theta\right)}{\sqrt{\beta_{1}}}=P_{1}+\sqrt{\beta_{1}} \theta=P_{1}+\Delta P,
\end{aligned}
$$

where $\beta_{1}$ and $\alpha_{1}$ are the Twiss parameters at the PSM location. From Eqs. (7) and (8), the kick angle in the normalized phase space is represented by

$$
\Delta P=\sqrt{\beta_{1}} \theta=-\frac{1}{2} \beta_{1}^{3 / 2} K_{2} X_{1}^{2} .
$$

On the other hand, the absolute value of the kick angle in the normalized phase space is obtained from geometric relations as shown in Fig. 2, 


$$
\begin{aligned}
|\Delta P| & =\left|P_{1}\right|-\left|P_{2}\right|=A_{\text {inj }}\left|\sin \phi_{1}\right|-\sqrt{A_{\text {red }}^{2}-X_{1}^{2}} \\
& =A_{\text {inj }}\left|\sin \phi_{1}\right|-\sqrt{A_{\text {red }}^{2}-A_{\text {inj }}^{2} \cos ^{2} \phi_{1}} .
\end{aligned}
$$

From geometric requirements, the absolute value of $X_{1}$ should be smaller than the radius of the inner circle,

$$
\left|X_{1}\right|<A_{\text {red }} .
$$

When the condition of Eq. (11) is satisfied, the square root part of Eq. (10) is always positive. From Eqs. (9) and (10), the field strength of the PSM becomes

$$
\left|K_{2}\right|=\frac{2\left(A_{\mathrm{inj}}\left|\sin \phi_{1}\right|-\sqrt{A_{\mathrm{red}}^{2}-A_{\mathrm{inj}}^{2} \cos ^{2} \phi_{1}}\right)}{\beta_{1}^{3 / 2} A_{\mathrm{inj}}^{2} \cos ^{2} \phi_{1}} .
$$

To obtain the smallest $K_{2}$ value, the PSM has to be installed at the location where the $\beta$ function has a large value and $\phi_{1}$ satisfies the condition $\cos \phi_{1} \sim \pm A_{\text {red }} / A_{\text {inj }}$, which is easily shown by the numerical calculation inputting specific $A_{\text {red }}$ and $A_{\text {inj }}$ values. We decided the location and $K_{2}$ of the PSM using Eq. (12) in Sec. III B.

Next, we consider the behavior of the stored beam in PSM injection. The stored beam predominantly produces SR light. For example, at the PF ring, the stored beam current is $450 \mathrm{~mA}$ and the number of filling bunches are 280 , while the injected beam current is typically around $0.02 \mathrm{~mA}$. The field strength of the PSM for the stored beam with finite beam size is almost zero in a parabolic magnetic field represented by Eq. (9) because the stored beam passes on the center of it, $X_{1}^{2} \sim 0$. It is expected that the beam oscillation of the stored beam is very small in the PSM injection.

\section{B. Difference from pulsed quadrupole injection}

Here, the PSM is compared with the pulsed quadrupole magnet (PQM). Figure 3 shows vertical integrated magnetic field distributions for both the PSM and the PQM,

$$
B_{y, \text { quad }}(x)=B_{\text {quad }}^{\prime} x, \quad B_{y, \text { sext }}(x)=\frac{1}{2} B_{\text {sext }}^{\prime \prime} x^{2},
$$

where $x$ is the horizontal displacement from the field center. When we provide the same field for the injected beam at a horizontal position of $x_{1}$ to both the magnets,

$$
B_{\text {quad }}^{\prime} x_{1}=\frac{1}{2} B_{\text {sext }}^{\prime \prime} x_{1}^{2},
$$

the field of the PSM at the position of $\Delta x$ equal to the stored beam size is weaker by a factor of $\Delta x / x_{1}$ than that of the PQM,

$$
\frac{B_{y, \text { sext }}(\Delta x)}{B_{y, \text { quad }}(\Delta x)}=\frac{\frac{1}{2} B_{\text {sext }}^{\prime \prime} \Delta x^{2}}{B_{\text {quad }}^{\prime} \Delta x}=\frac{\Delta x}{x_{1}},
$$

and the field gradient is also weaker by

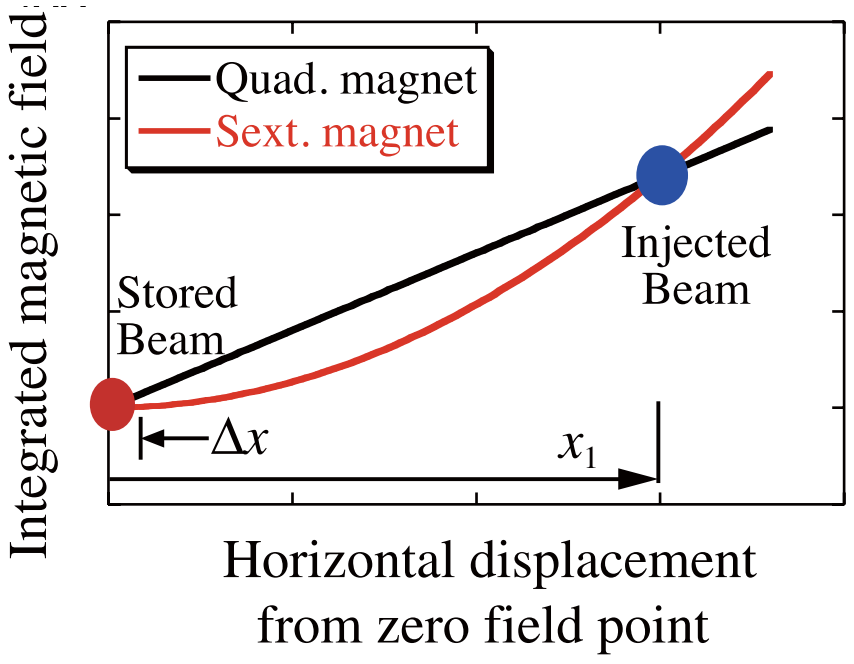

FIG. 3. (Color) Integrated magnetic fields of a PSM and a PQM as a function of horizontal displacement from the zero field point. To compare effects of the magnetic fields of the PSM and PQM on a stored beam, the field strengths of both magnets have the same value at the position of an injected beam orbit.

$$
\frac{B_{y, \text { sext }}^{\prime}(\Delta x)}{B_{y, \text { quad }}^{\prime}(\Delta x)}=\frac{B_{\text {sext }}^{\prime \prime} \Delta x}{B_{\text {quad }}^{\prime}}=\frac{2 \Delta x}{x_{1}}
$$

From this result, the PSM injection has the advantage that both the field gradient and the field strength on the stored beam are smaller than those of the PQM. For example, the injected beam position and the horizontal beam size of the $\mathrm{PSM}$ at the PF ring are $x_{1}=15 \mathrm{~mm}$ and $\Delta x=0.7 \mathrm{~mm}$, respectively; the integrated magnetic field of the PSM for the stored beam becomes $95 \%$ smaller than that of the PQM; and the field gradient becomes $90 \%$ smaller.

The PQM injection method was already examined at the photon factory advanced ring (PF-AR), which is a $6.5 \mathrm{GeV}$ electron storage ring dedicated as a SR source [6]. Figure 4 shows the turn-by-turn stored beam profiles just after the beam injection with the pulsed bump; the PQM was measured by a fast gated camera. The large horizontal coherent dipole oscillation of the stored beam could be seen in the pulsed bump injection. The PQM injection system reduced

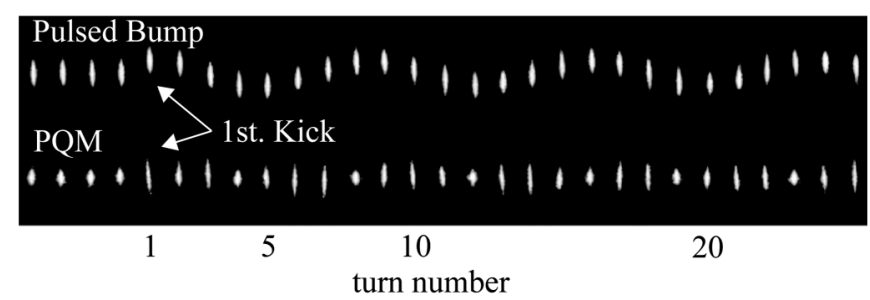

FIG. 4. Turn-by-turn stored beam profiles in pulsed bump injection (upper figure) and PQM injection (lower figure) monitored by a fast gated camera at the PF-AR. The horizontal axis represents a turn number and the vertical axis represents a horizontal direction. 
the dipole oscillation compared with the pulsed bump injection. However, a beam profile modulation of the stored beam (like a quadrupole mode oscillation) was clearly observed in the PQM injection as seen in the lower figure. The source of the modulation is the magnetic field of the PQM around the field center through which the stored beam passes. It is also expected that the beam profile modulation is much reduced by the PSM injection because of its smaller field on the stored beam.

\section{APPLICATION OF PSM INJECTION TO THE PF RING}

\section{A. PF ring parameters}

We designed the PSM injection system for an application to the PF ring, which is a $2.5 \mathrm{GeV}$ electron storage ring dedicated as a SR source. The main parameters of the PF ring are listed in Table I. The injected beam passes through a horizontal position of $27 \mathrm{~mm}$ from the central orbit of the ring at the exit of the septum magnet (injection point). The square of the injection amplitude, $A_{\mathrm{inj}}^{2}$ in Eq. (2), is $67 \mathrm{~mm}$ mrad with the horizontal Twiss parameters of $\beta_{0}=11.2 \mathrm{~m}$ and $\alpha_{0}=-1.20$ and injection angle of $x_{0}^{\prime}=2.5 \mathrm{mrad}$ (the positive kick angle represents the kick to the outside of the ring). The ring acceptance is about $40 \mathrm{~mm}$ mrad. Considering the margin of error, the square of the reduced injection amplitude $A_{\text {red }}^{2}$ was set to $20 \mathrm{~mm}$ mrad.

\section{B. Single particle simulation}

First, we had to find an installation point of the PSM where the coherent dipole oscillation of the injected beam could be effectively reduced. The SAD code, a tracking simulation code developed in KEK [7], was used to simulate the PSM injection. The location was searched using Eq. (12) with $x_{0}=27 \mathrm{~mm}$ and $A_{\text {red }}^{2}=20 \mathrm{~mm} \mathrm{mrad}$.

Candidates for the PSM installation points around the downstream side of the injection point were listed. Each candidate was required to have free space of at least $1 \mathrm{~m}$.

TABLE I. Main parameters of the PF ring.

\begin{tabular}{lcc}
\hline \hline Parameter & Symbol & Value \\
\hline Beam energy & $E$ & $2.5 \mathrm{GeV}$ \\
Circumference & $C$ & $187 \mathrm{~m}$ \\
Betatron tunes & $\nu_{x}, \nu_{y}$ & $9.60,5.28$ \\
Revolution period & $\tau$ & $624 \mathrm{~ns}$ \\
Horizontal emittance & $\varepsilon_{x}$ & $35.8 \mathrm{~nm} \mathrm{rad}$ \\
Vertical emittance & $\varepsilon_{y}$ & $0.358 \mathrm{~nm} \mathrm{rad}$ \\
Energy spread & $\sigma_{\varepsilon}$ & $7.3 \times 10^{-4}$ \\
Bunch length & $\sigma_{z}$ & $9.6 \mathrm{~mm}$ \\
Horizontal beam size & $\sigma_{x}$ & $0.674 \mathrm{~mm}^{\mathrm{a}}$ \\
Vertical beam size & $\sigma_{y}$ & $0.048 \mathrm{~mm}^{\mathrm{a}}$ \\
Radiation damping time & $\tau_{x}, \tau_{y}, \tau_{z}$, & $7.8,7.8,3.9 \mathrm{~ms}$ \\
\hline \hline
\end{tabular}

${ }^{\mathrm{a}}$ At the center of the PSM where the horizontal and vertical betatron functions are 12.66 and $6.57 \mathrm{~m}$, respectively.
There were six candidates labeled PSM01 to PSM06 as shown in Fig. 5. The betatron functions and the phase angles at the PSM locations are listed in Table II. Figure 6 shows the calculated field strengths for each

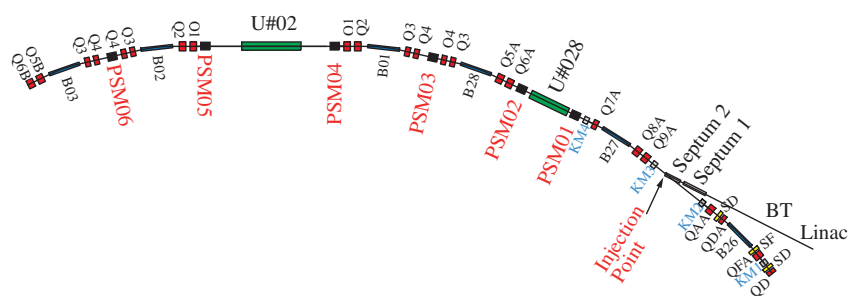

FIG. 5. (Color) A lattice configuration around the injection point at the PF ring. Six candidates for the PSM location are shown. The locations are free, straight sections. The labels B, Q, and S represent bending, quadrupole, and sextupole magnets, respectively. KM1-KM4 indicate kicker magnets for the pulsed bump injection.

TABLE II. Horizontal betatron functions and phase angles at the candidate locations of the PSM.

\begin{tabular}{lcc}
\hline \hline Location & $\beta_{x}(\mathrm{~m})$ & $\phi_{1}(\mathrm{deg})$ \\
\hline PSM01 & 4.51 & 158.9 \\
PSM02 & 12.45 & 179.0 \\
PSM03 & 5.30 & 313.5 \\
PSM04 & 12.66 & $100.2+360$ \\
PSM05 & 12.66 & $118.1+360$ \\
PSM06 & 5.43 & $241.6+360$ \\
\hline \hline
\end{tabular}

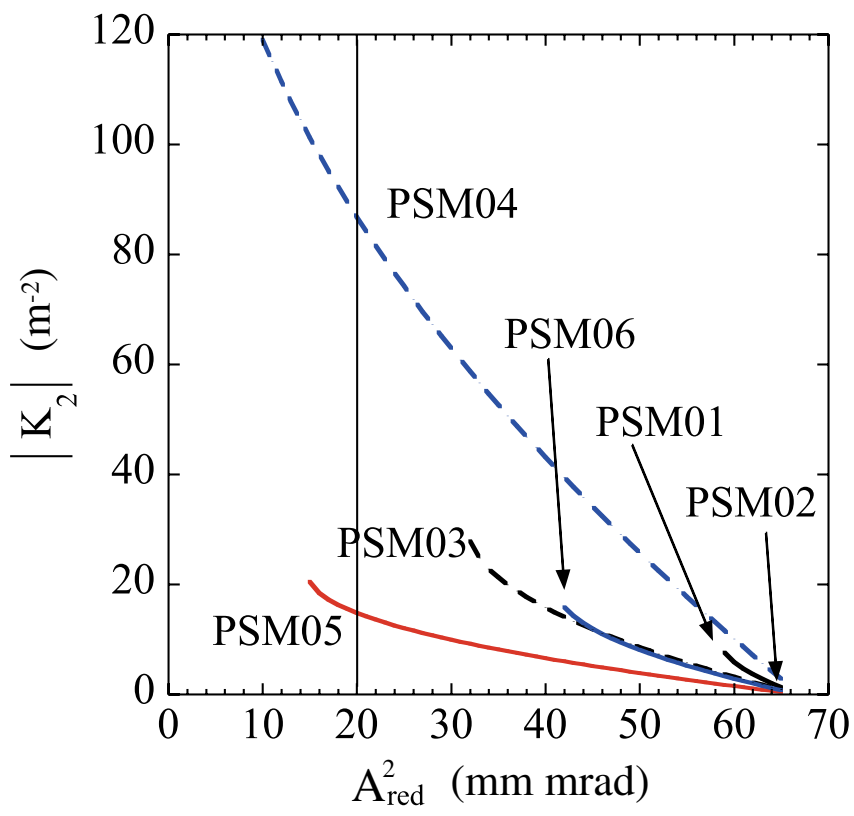

FIG. 6. (Color) PSM field strengths at the PSM locations as a function of the square of the reduced injection amplitude $\left(A_{\text {red }}^{2}\right)$. In the calculations, the injection angle of the injected beam is $x_{0}^{\prime}=2.5 \mathrm{mrad}$. 
candidate with an injection angle of $x_{0}^{\prime}=2.5 \mathrm{mrad}$. For PSM02, the condition of (11) is only satisfied in the region of $A_{\text {red }} \sim A_{\text {inj }}$, because the phase angle is about 180 degrees. For PSM01, PMS03, and PSM06, the solution of $K_{2}$ does not exist at $A_{\text {red }}^{2}=20 \mathrm{~mm}$ mrad. PSM04 needs to have a very large field strength of the PSM. In these conditions, Fig. 6 shows that the best location is PSM05. Thus, we selected PSM05 as the PSM installation location, which is a free space downstream of the long straight section of the undulator \#02 (U\#02) and is located about $30 \mathrm{~m}$ away from the injection point.

Fine-tuning of the PSM field strength at PSM05 was carried out. Once the optics of the ring are decided, the phase advance $\psi$ from the injection point to the PSM is fixed, and the phase angle $\phi_{1}$ at the PSM can be controlled by the injection angle $\left(x_{0}^{\prime}\right)$,

$$
\phi_{1}=\psi+\arctan \frac{-P_{0}}{X_{0}}=\psi+\arctan \left(-\alpha_{0}-\beta_{0} \frac{x_{0}^{\prime}}{x_{0}}\right) .
$$

When the injected beam is kicked toward the outside direction $\left(x_{0}^{\prime}>0\right)$ in Fig. 2, the phase angle at the PSM location, $\phi_{1}$, slightly decreases as a result of the increase of $P_{0}$. Figure 7 shows the field strength of PSM05 as a function of the reduced injection amplitude and the injection angle. The injection angle should be more than $2.3 \mathrm{mrad}$ to achieve the square of the reduced injection amplitude of $20 \mathrm{~mm}$ mrad. $K_{2}$ has the smallest value of $13 \mathrm{~m}^{-2}$ around the injection angle of $2.4 \mathrm{mrad}$ with $A_{\mathrm{red}}^{2}=$

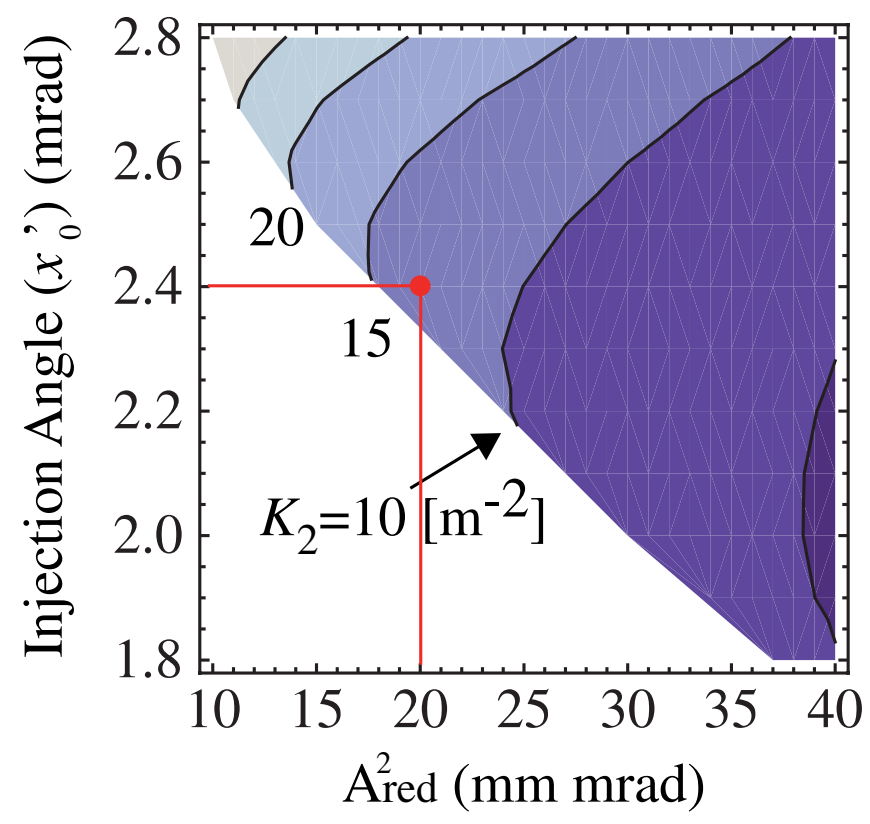

FIG. 7. (Color) The field strength $K_{2}$ of the PSM05 is shown as a function of the square of the reduced injection amplitude $A_{\text {red }}^{2}$ and the injection angle $x_{0}^{\prime}$. The white area has no solution of $K_{2}$ because the condition of (11) is not satisfied. Calculations were made with $x_{0}=27 \mathrm{~mm}$ and $A_{\text {red }}^{2}=20 \mathrm{~mm}$ mrad using Eq. (12).

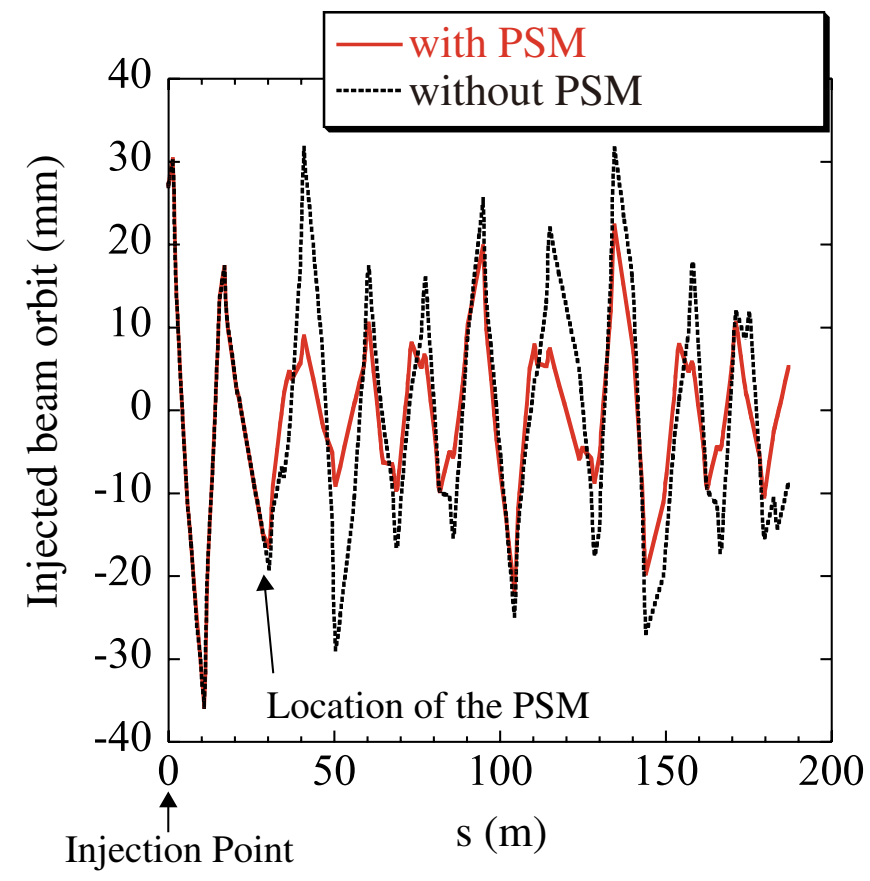

FIG. 8. (Color) Horizontal orbit of the injected beam at the PF ring with (red) and without (black) a PSM. The orbit displays the first circulation of the beam.

$20 \mathrm{~mm} \mathrm{mrad}$. This value corresponds to the field gradient $B^{\prime \prime}$ of $416 \mathrm{~T} / \mathrm{m}^{2}$ with a magnet core length of $0.3 \mathrm{~m}$ and the integrated field $B \ell$ of 120 Gauss $\mathrm{m}$ at a horizontal position of $15 \mathrm{~mm}$. Figure 8 shows the center motion of the injected beam at its first turn with $K_{2}=13 \mathrm{~m}^{-2}$.

\section{Multiparticle simulation}

In the previous section, we simulated the center motion of an injected beam. In this section, we calculate the capture efficiency of the PSM injection using a multiparticle tracking defined by

$$
\varepsilon_{\text {cap }}=\frac{N_{\text {res }}}{N_{\text {ini }}},
$$

where $N_{\text {ini }}$ is the number of initial particles and $N_{\text {res }}$ is the number of residual particles after 100 turns. A sixdimensional Gaussian distribution is used for the initial beam.

Since a revolution period of the PF ring is about $0.6 \mu \mathrm{s}$, a half-sine pulse with a full width of less than $1.2 \mu \mathrm{s}$ is preferable for the PSM excitation pulse. Using this pulse, the capture efficiency becomes more than $90 \%$ with $K_{2}=$ $13 \mathrm{~m}^{-2}$ and $x_{0}^{\prime}=2.0 \mathrm{mrad}$ (see one-turn injection in Fig. 9).

Unfortunately, for economical reasons, we could not provide a pulsed power supply with a pulse width of less than $1.2 \mu \mathrm{s}$, and hence, employed a second-hand pulsed power supply with a full width of $2.4 \mu \mathrm{s}$. Although that power supply has sufficient capacity for the charging volt- 


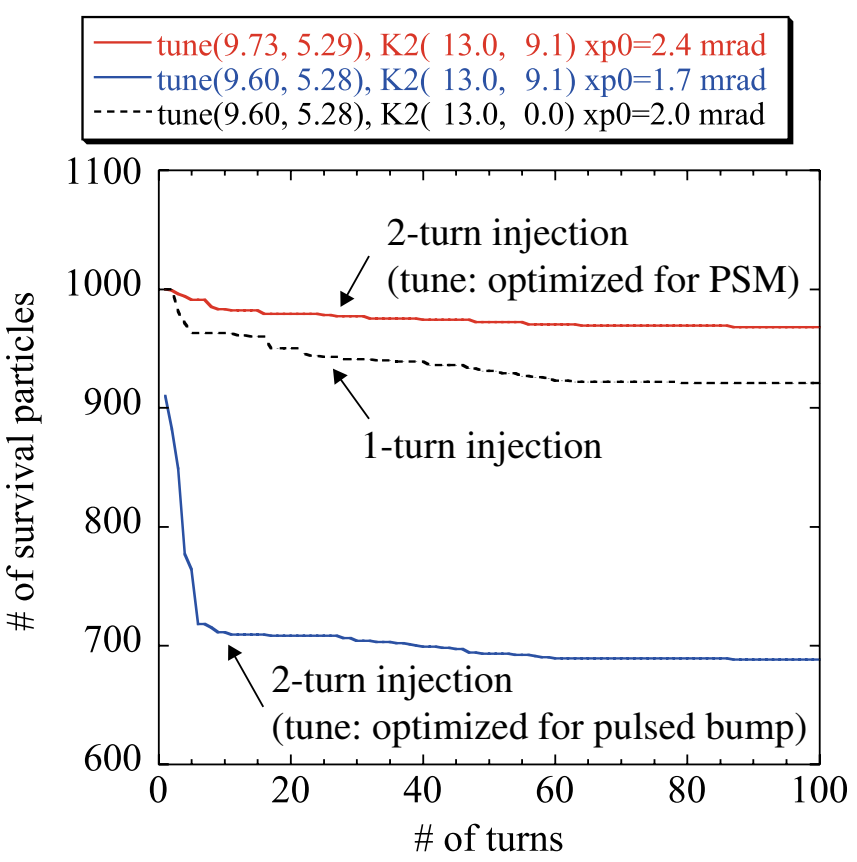

FIG. 9. (Color) The number of survival particles in the PSM injection plotted as a function of turn number. Parameters in the legend show the operating tune $\left(\nu_{x}, \nu_{y}\right)$, the field strength of the PSM (1st kick, 2nd kick), and the injection angle $\left(x_{0}^{\prime}\right)$.

age and the output current, the injected beam inevitably receives an additional kick from the PSM due to the longer pulse width. We call this injection scheme "two-turn injection." This problem is not general to the PSM injection but a specific problem caused by the longer injection pulse of the employed pulsed power supply.

In the two-turn injection, the first kick is adjusted at the peak of the half-sine waveform and the second kick is provided after $0.624 \mu \mathrm{s}$ from the peak with a strength which is 0.3 times that of the first kick. It is essential to choose a proper betatron tune in order to suppress the increase of coherent dipole oscillation of the injected beam due to the second kick. We estimated the effect of the second kick in the beam injection, changing the betatron tune and the injection angle. The capture efficiency was about $70 \%$ at the tune of $\left(\nu_{x}, \nu_{y}\right)=(9.60,5.28)$ which is the normal operating point of the PF ring and optimized for the conventional pulsed bump injection as shown in Fig. 9. After the tune was optimized to $\left(\nu_{x}, \nu_{y}\right)=$ $(9.73,5.29)$ for the PSM two-turn injection, the capture efficiency could exceed $95 \%$ on the condition of $K_{2}=$ $13 \mathrm{~m}^{-2}$ at the first kick $\left(9.1 \mathrm{~m}^{-2}\right.$ at the second kick) and the injection angle $=2.4 \mathrm{mrad}$. However, we used the operating tune of $\left(\nu_{x}, \nu_{y}\right)=(9.60,5.28)$ and did not change it to the optimized tune for the PSM injection in the following calculations and beam injection experiments.

Figure 10 shows the capture efficiency as a function of the injection angle. The capture efficiency strongly depends on the injection angle. The injection angle of about

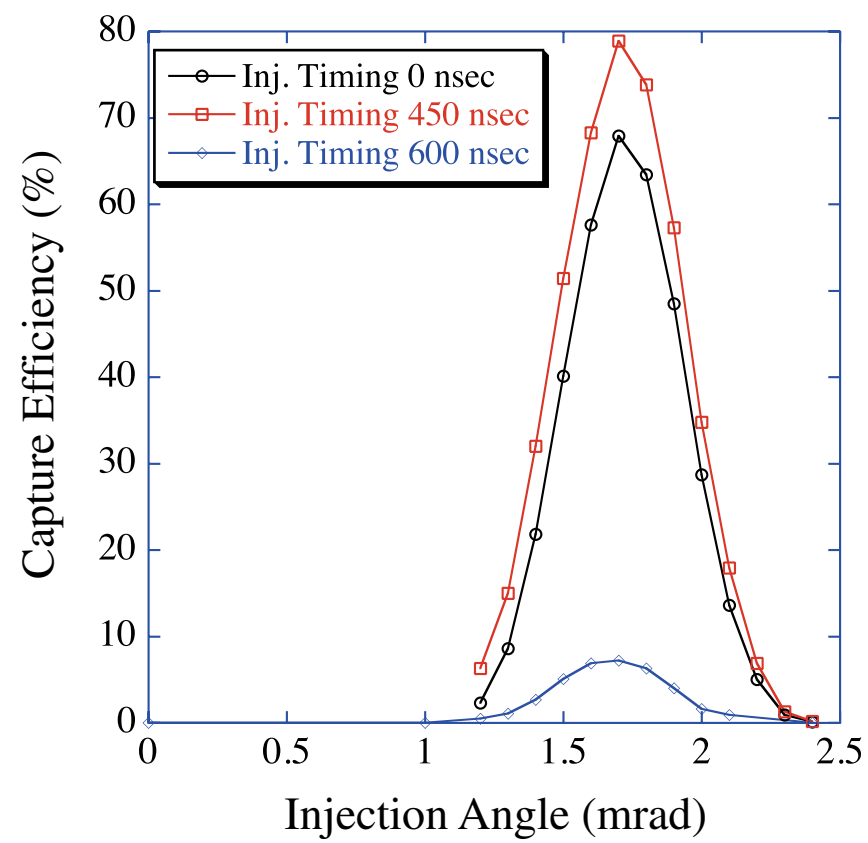

FIG. 10. (Color) Capture efficiency as a function of the injection angle. The circles, squares, and diamonds show the efficiencies when the injection timings are 0,450 , and $600 \mathrm{~ns}$, respectively. The operating tune is $\left(\nu_{x}, \nu_{y}\right)=(9.60,5.28)$.

$1.7 \mathrm{mrad}$ can realize the capture efficiency of about $70 \%$. Figure 11 shows the capture efficiency as a function of the injection timing. The timing dependency of the capture

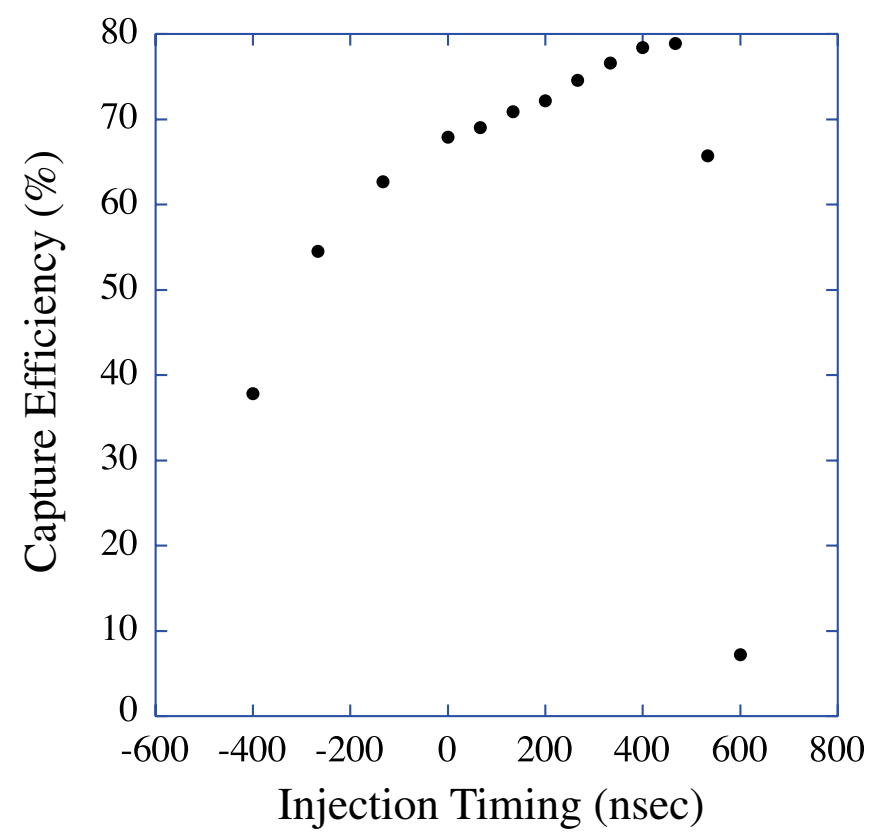

FIG. 11. (Color) Capture efficiency as a function of the injection timing with the injection angle of $1.7 \mathrm{mrad}$. The "zero" denotes that the injected beam comes at the peak of the PSM's excitation. The positive value means that the injected beam is delayed compared with the pulse peak. The betatron tune is $\left(\nu_{x}, \nu_{y}\right)=$ (9.60, 5.28). 
efficiency is rather gentler than that of the injection angle. In a wide range of the injection timing from -200 to $500 \mathrm{~ns}$, the capture efficiency exceeds $60 \%$ with the injection angle of $1.7 \mathrm{mrad}$. From the multiparticle tracking simulation, we confirmed that the two-turn injection worked well in the normal operating tune of the PF ring, though the capture efficiency was reduced by about $20 \%-$ $30 \%$ compared with the one-turn injection or two-turn injection in the optimized tune.

\section{PSM INJECTION SYSTEM}

\section{A. Magnet design}

The main parameters of the PSM are listed in Table III. The bore diameter of the PSM was determined first so that the PSM did not limit the physical aperture of the PF ring. Next, to obtain as strong a magnetic field as possible, a round magnetic pole surface was adopted as shown in Fig. 12. This pole shape has the strongest field among several different pole shapes. Since the PSM kicks an injected beam only twice, even in a two-turn injection, it is not essential to have a pure sextupole magnetic field. The magnetic field distribution calculated by the twodimensional magnetic field calculation code POISSON [8] is shown in Fig. 12. This shape can realize a sufficient magnetic field for the injected beam. To reduce eddy current effects, the magnet was made of 0.15 -mm-thick laminated silicon steel sheets. The coil was formed by a one-turn copper bar with a diameter of $15 \mathrm{~mm}$. The PSM is air cooled without a blower.

\section{B. Field measurement}

The designed pulsed sextupole magnet was fabricated (see Fig. 13) and the pulsed magnetic field was then measured by two kinds of search coils, a short rectangular coil of $5 \mathrm{~mm} \times 5 \mathrm{~mm}$ and a long rectangular coil of $5 \mathrm{~mm} \times 600 \mathrm{~mm}$. The search coils were fixed to a 3D movable stage. The stage was remotely controlled by a stepping motor with a precision of $0.2 \mu \mathrm{m} /$ pulse for the horizontal $(X)$ and the longitudinal $(Z)$ directions and $1 \mu \mathrm{m} /$ pulse for the vertical $(Y)$ direction. The pulsed power supply has a maximum charging voltage of $40 \mathrm{kV}$

TABLE III. Main parameters of the PSM system.

\begin{tabular}{lc}
\hline \hline Parameter & Value \\
\hline Core length & $300 \mathrm{~mm}$ \\
Bore diameter & $66 \mathrm{~mm}$ \\
Number of coil turns & 1 \\
Integrated magnetic field at $x=15 \mathrm{~mm}$ & $120 \mathrm{Gauss}$ \\
Peak current & $3000 \mathrm{~A}$ \\
Inductance & $4.3 \mu \mathrm{H}$ \\
Pulse width & $1.2(2.4)^{\mathrm{a}} \mu \mathrm{s}$ \\
\hline \hline
\end{tabular}

${ }^{\mathrm{a}}$ The design value is $1.2 \mu \mathrm{s}$, but prepared power supply had $2.4 \mu \mathrm{s}$.

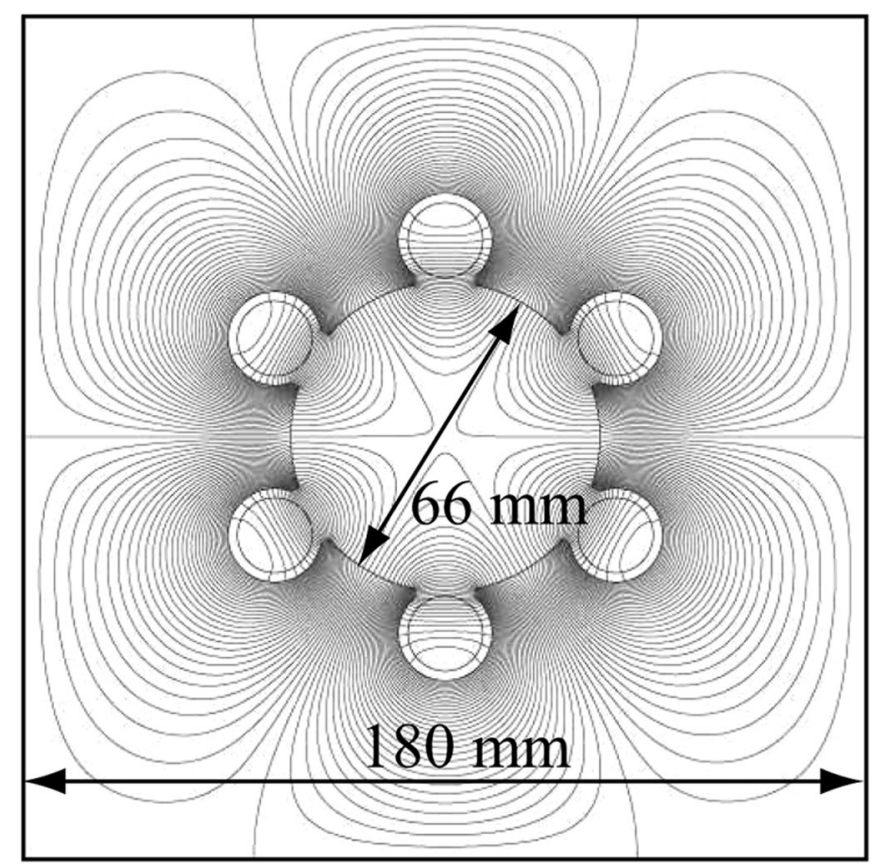

FIG. 12. Cross-sectional view and two-dimensional magnetic field distribution of the PSM. The bore diameter is $66 \mathrm{~mm}$. The coil is a one-turn copper bar with a diameter of $15 \mathrm{~mm}$.

and produces an output current of a half-sine pulse shape with a full width of $2.4 \mu$ s. Since the total inductance of the PSM was $4.3 \mu \mathrm{H}$, the required charging voltage of $16.7 \mathrm{kV}$ could be easily achieved for a peak current of $3000 \mathrm{~A}$, which corresponds to a field strength of $K_{2}=$ $13 \mathrm{~m}^{-2}$. The repetition frequency is available up to $12.5 \mathrm{~Hz}$. In order to reduce the noise that was mainly generated by the mismatch of the impedance, a low-pass filter of $2 \mathrm{MHz}$ and a ferrite core was added to the signal line. Figure 14 shows the typical output waveforms of the search coils measured at the charging voltage of $16.7 \mathrm{kV}$.

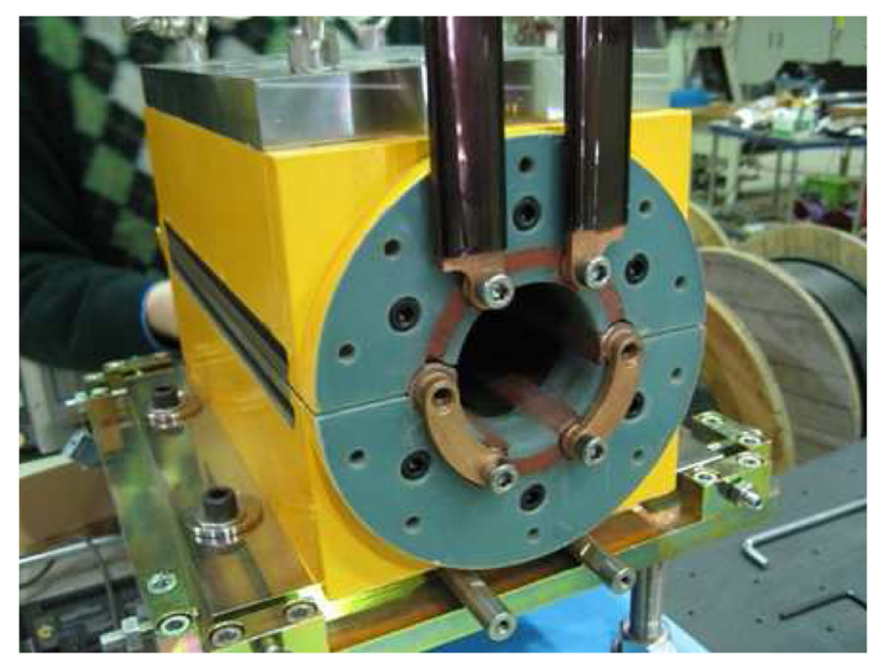

FIG. 13. (Color) Front view of the PSM. The glass epoxy board (green) and the epoxy resin (brown) are used for insulation. 


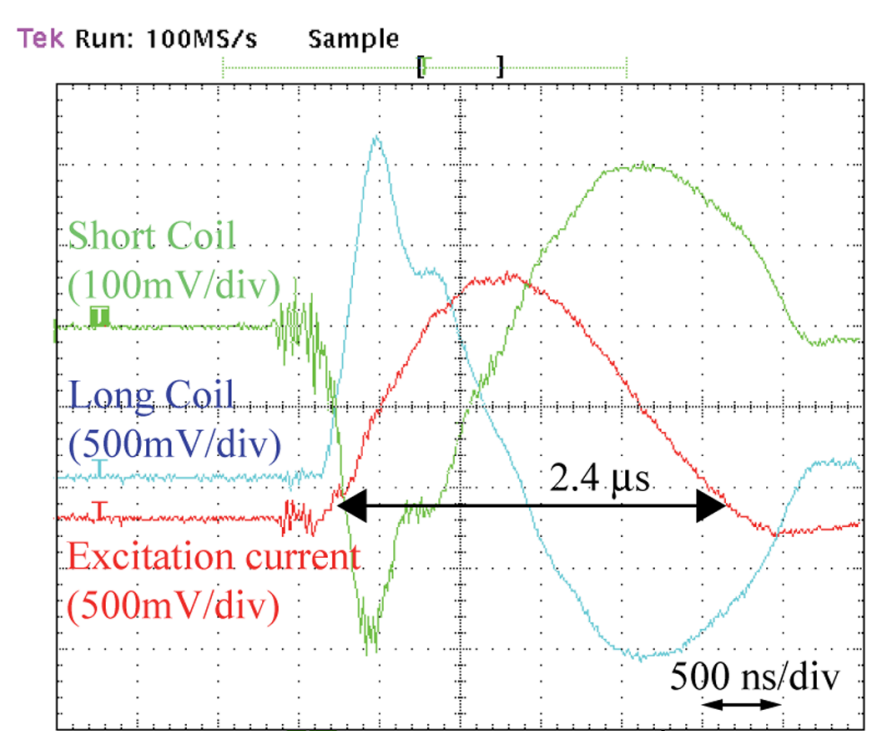

FIG. 14. (Color) Typical output waveforms of search coils measured at a charging voltage of $16.7 \mathrm{kV}$. The red solid line represents an excitation current of the PSM. Green and cyan solid lines represent output signals of the short coil and the long coil, respectively. The search coils are placed at a horizontal location of $15 \mathrm{~mm}$ from the magnetic pole center of the PSM.

The magnetic field was evaluated by integrating the output voltage of the search coils. Figure 15 compares the measured horizontal distribution of the integrated field at a peak current of $3000 \mathrm{~A}$ with the calculated result of the POISSON code. The horizontal distribution of the measured integrated field showed a good parabolic shape and good agreement with the calculated result. The field strength exceeded the required integrated magnetic field of

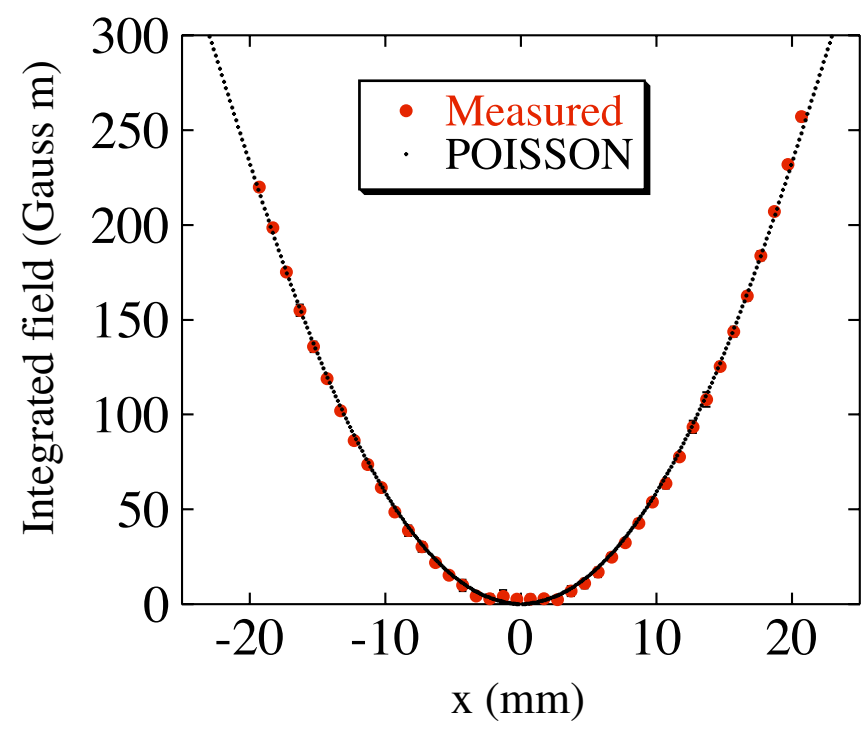

FIG. 15. (Color) Horizontal distribution of the integrated magnetic field of the PSM. Solid circles (red) show the measured value, and the dotted line (black) shows the calculated result of the POISSON code.
120 Gauss $\mathrm{m}$ at a horizontal position of $15 \mathrm{~mm}$ and was nearly zero at the center of the PSM. The fluctuation of the field at $x=15 \mathrm{~mm}$ was $5 \times 10^{-3}(\mathrm{rms})$. The result of the POISSON was calculated considering that the measured effective length of the PSM is $320 \mathrm{~mm}$.

\section{Vacuum chamber}

The PSM vacuum chamber was made of a ceramic $\left(\mathrm{Al}_{2} \mathrm{O}_{3}\right)$ to reduce eddy currents which cause distortion and delay of the pulsed magnetic field. The thickness of the ceramic was determined to be $3 \mathrm{~mm}$ considering the structural strength of the ceramic chamber. The inner diameter was decided to be $59 \mathrm{~mm}$ by the beam stay clear with $5 \mathrm{~mm}$ margin. A clearance of $0.5 \mathrm{~mm}$ was secured between the ceramic chamber and the magnetic pole. The inner side was coated with a titanium layer of $3 \mu \mathrm{m}$. In addition, a $\mathrm{Cu}$ absorber with water cooling was attached upstream of the chamber to protect the ceramic from SR generated in an upstream bending magnet.

\section{Installation of the PSM}

The PSM was installed in the spring of 2008. Figure 16 shows a photograph of the PSM installed downstream of the U\#02. The PSM was aligned using a telescope for the horizontal and vertical directions and a steel gauge for the longitudinal direction. The reference points were quadrupole magnets placed at both ends of the U\#02 straight section. Horizontal and vertical alignment errors of the PSM were $\pm 100 \mu \mathrm{m}$. The longitudinal alignment error was $\pm 500 \mu \mathrm{m}$.

\section{E. Timing tuning}

Charge and discharge of a pulsed power supply are controlled by external triggers which are provided by the KEK linac. The discharge trigger coincides with the linac

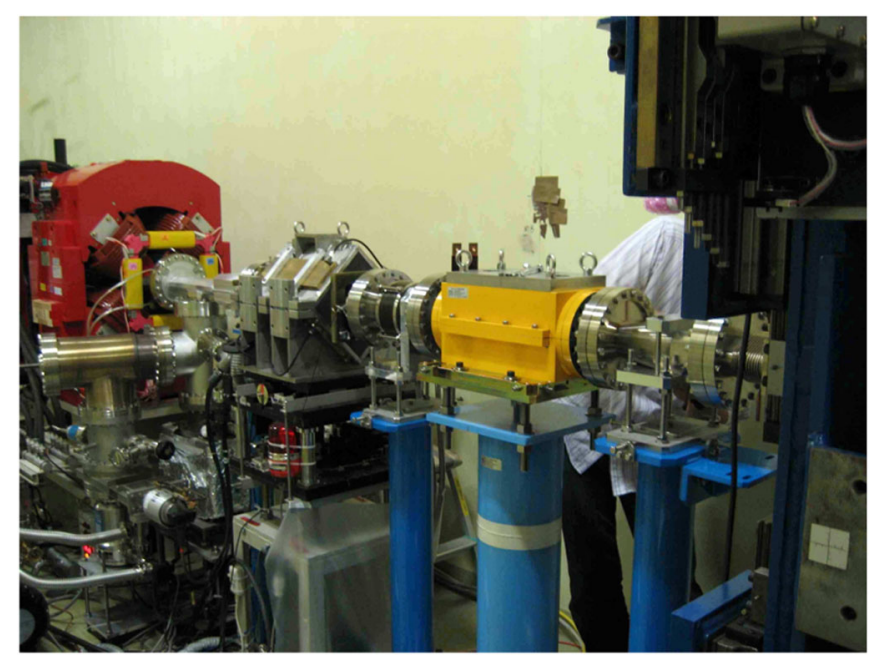

FIG. 16. (Color) Photograph of the PSM (yellow) installed in the $\mathrm{PF}$ ring. 


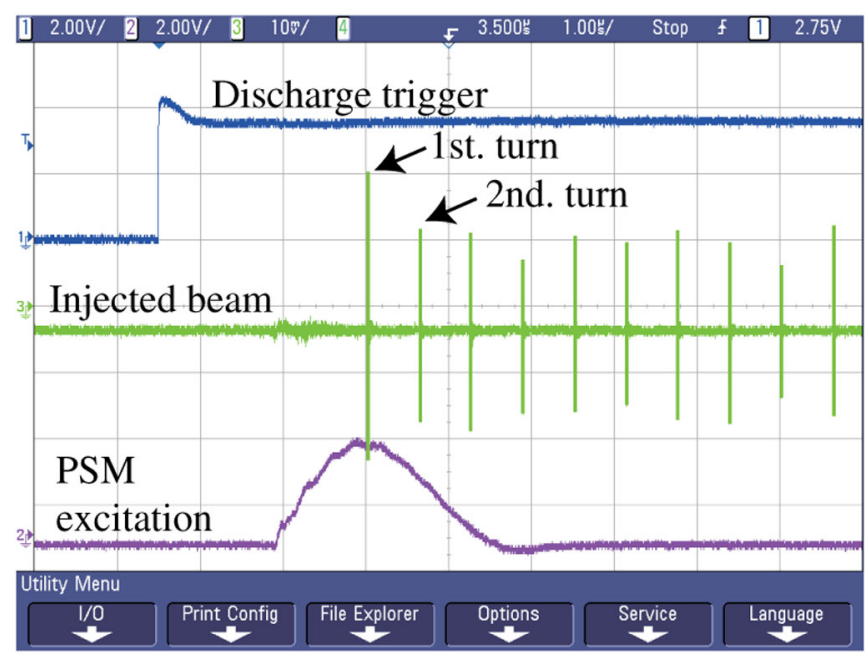

FIG. 17. (Color) Typical waveforms of a discharge trigger (blue), an injected beam signal from a wall current monitor of the ring (green), and a PSM excitation signal from a current transformer of the pulsed power supply (purple) measured by a digital oscilloscope.

frequency of $2856 \mathrm{MHz}$ and a revolution frequency of the $\mathrm{PF}$ ring of $1.60 \mathrm{MHz}$, and its timing jitter is about $\pm 175 \mathrm{ps}$. Because the pulsed power supply needs $10 \mathrm{msec}$ for charge-up, the charge trigger is generated from the discharge trigger so as to come about $10 \mathrm{msec}$ before the discharge trigger for any repetition frequency.

Precise tuning of the discharge trigger, which determines the temporal relation between excitation of the PSM and beam injection, was performed using an injected beam in single-bunch mode. The timing of the injected beam was monitored using a wall current monitor placed downstream of the bending magnet B03 in Fig. 5. Figure 17 shows the timing signal of the injected beam and the PSM excitation pulse. The tuning was conducted after turning off the radio-frequency cavity so that the injected beam did not accumulate. The timing of the PSM first kick to the injected beam was set on the peak of the PSM excitation pulse.

\section{BEAM INJECTION EXPERIMENTS}

\section{A. Multibunch injection}

The first beam injection experiment with the PSM was conducted in May 2008. Figure 18 shows the progress of the stored beam current and the injection rate with multibunch injection in the first injection experiment. At the first stage, injection was carried out at a repetition frequency of $5 \mathrm{~Hz}$. The beam transport, including the septum magnet, was tuned so as to maximize the injection rate. After tuning, the frequency was increased to $12.5 \mathrm{~Hz}$. On the way to $450 \mathrm{~mA}$, a pulsed bump injection with a repetition rate of $25 \mathrm{~Hz}$ was employed to compare the injection rates [9]. When the beam current exceeded $400 \mathrm{~mA}$, the PSM

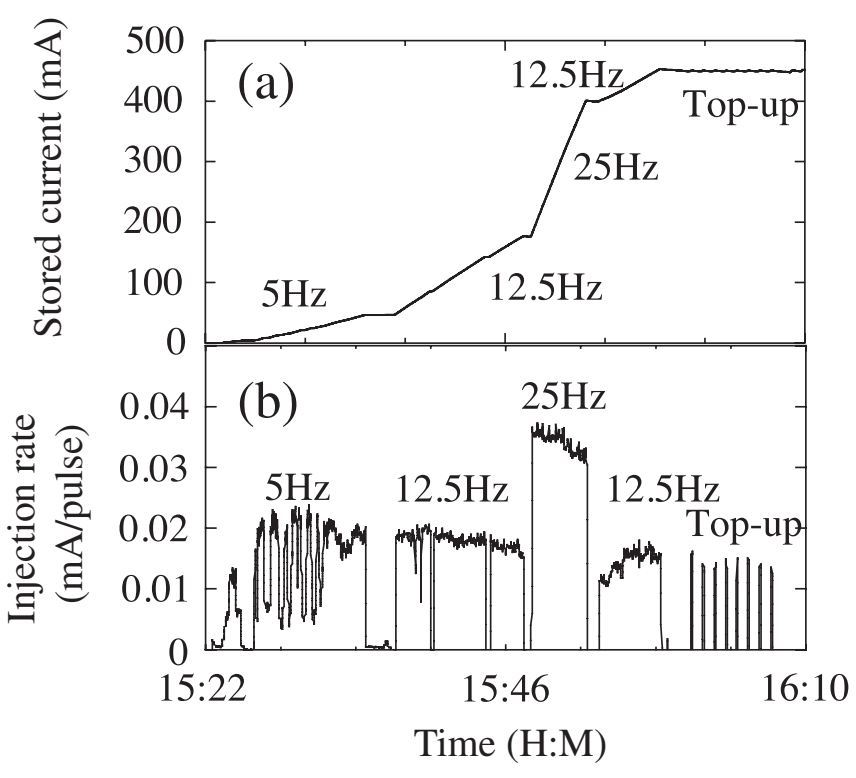

FIG. 18. Progress of the stored beam current is shown in (a) and the injection rate in (b). The beam was injected using the PSM injection ( 5 and $12.5 \mathrm{~Hz}$ ) and the pulsed bump injection $(25 \mathrm{~Hz})$. Since the injection rate is represented by the injected beam current per an injection pulse, the injection rate can be compared directly between two injection modes.

injection was resumed. Even at high currents over $400 \mathrm{~mA}$, the PSM injection worked well, though the rate of the PSM was lower by about a half than that of the pulsed bump injection.

\section{B. Top-up injection}

Top-up injection using the PSM injection was examined at $450 \mathrm{~mA}$. The stability of the stored beam current during the top-up injection was measured. The operating conditions were: (i) The PSM injection started with $1 \mathrm{~Hz}$ when the stored beam current was less than $450.00 \mathrm{~mA}$; (ii) the injection stopped if the beam current exceeded $450.05 \mathrm{~mA}$; (iii) the stored beam current was monitored with $2 \mathrm{~Hz}$. Figure 19 shows the stored beam current during the top-up injection for two hours; the small window is an expanded view of 10 minutes. The injection rate was $0.005 \sim$ $0.02 \mathrm{~mA} /$ pulse. Top-up injection with the PSM achieved good stability of less than $0.02 \%$ in peak to peak for two hours.

\section{Stability of stored beam position}

The greatest advantage of the PSM injection is that the beam oscillation of the stored beam during the injection can be very small. We compared the stored beam oscillation in the PSM injection with that in the pulsed bump injection using a turn-by-turn beam position monitor (BPM). The stored beam oscillation was measured in the single-bunch mode to monitor it precisely, and the stored beam current was set to around $30 \mathrm{~mA}$. Figure 20 shows 


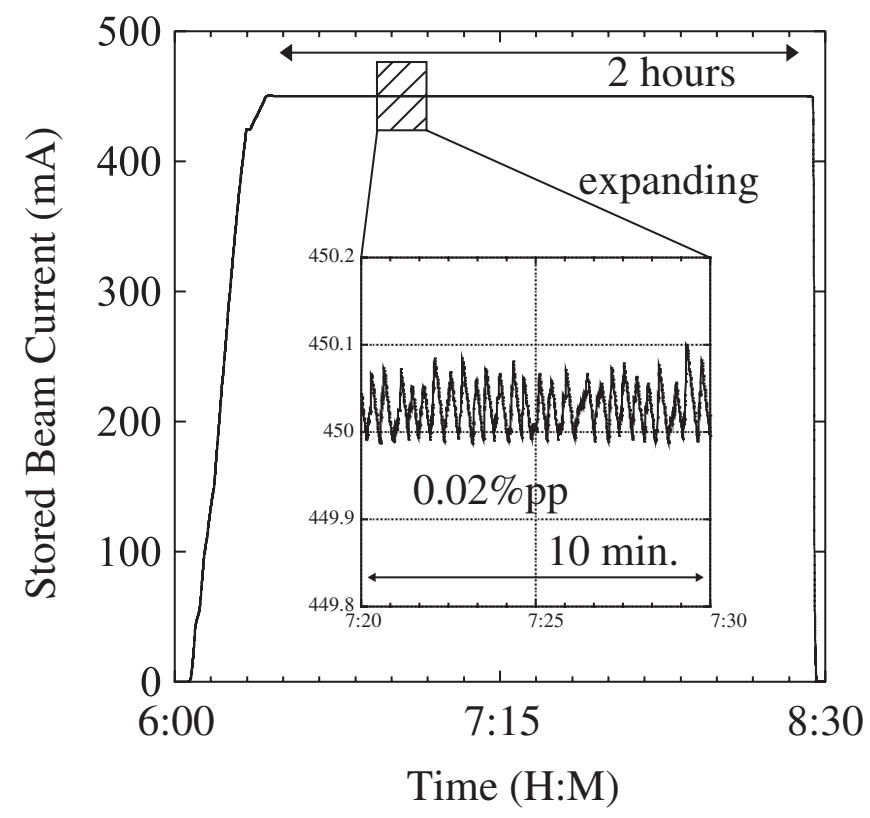

FIG. 19. Stability of the stored beam current for 2 hours during the top-up injection with the PSM.

the horizontal and vertical coherent dipole oscillations of the stored beam just after the injection. During the pulsed bump injection, large dipole oscillations in both the horizontal and vertical directions were observed as shown in Figs. 20(a) and 20(b). The oscillations were generated due to the mismatch of the four pulsed kicker magnets. The amplitudes of the oscillations were about $850 \mu \mathrm{m}$ and $130 \mu \mathrm{m}$ at the maximum in the horizontal and vertical direction, respectively. On the other hand, as shown in Figs. 20(c) and 20(d), the maximum amplitudes during PSM injection were reduced to about 180 and $40 \mu \mathrm{m}$ in the horizontal and vertical direction, respectively. The vertical oscillation corresponded to almost the noise level of the turn-by-turn BPM system. The coherent dipole oscillations of the stored beam in the PSM injection were sufficiently reduced, as compared with those in the pulsed bump injection.

\section{Stability of photon beam intensity}

To show that the top-up injection with the PSM is also effective for high-quality SR experiments, we measured the stability of the photon intensity at three beam lines, BL5A, BL-14A, and BL-17A, in multibunch mode at $450 \mathrm{~mA}$. The pulsed bump injection and the PSM injection were individually operated at $1 \mathrm{~Hz}$ without an injected beam so as to monitor the effect on the stored beam directly. The effect of the injected beam on the photon intensity was estimated from the ratio of the number of particles in the injected beam to the stored beam to be $\sim 2 \%-3 \%$ at maximum in the top-up injection.

BL-14A has a vertical superconducting wiggler, and its photon intensity stability is very sensitive to the horizontal beam oscillation. The photon intensity was measured with a PIN photodiode after focusing by a toroidal mirror and passing through a pinhole with a diameter of $0.8 \mathrm{~mm}$. Figure 21 shows the measurement result. If the photon intensity during the beam injection is not changed, the signal from the photodiode has a constant value around unity. However, the photon intensity had a $1 \mathrm{~Hz}$ spike train,
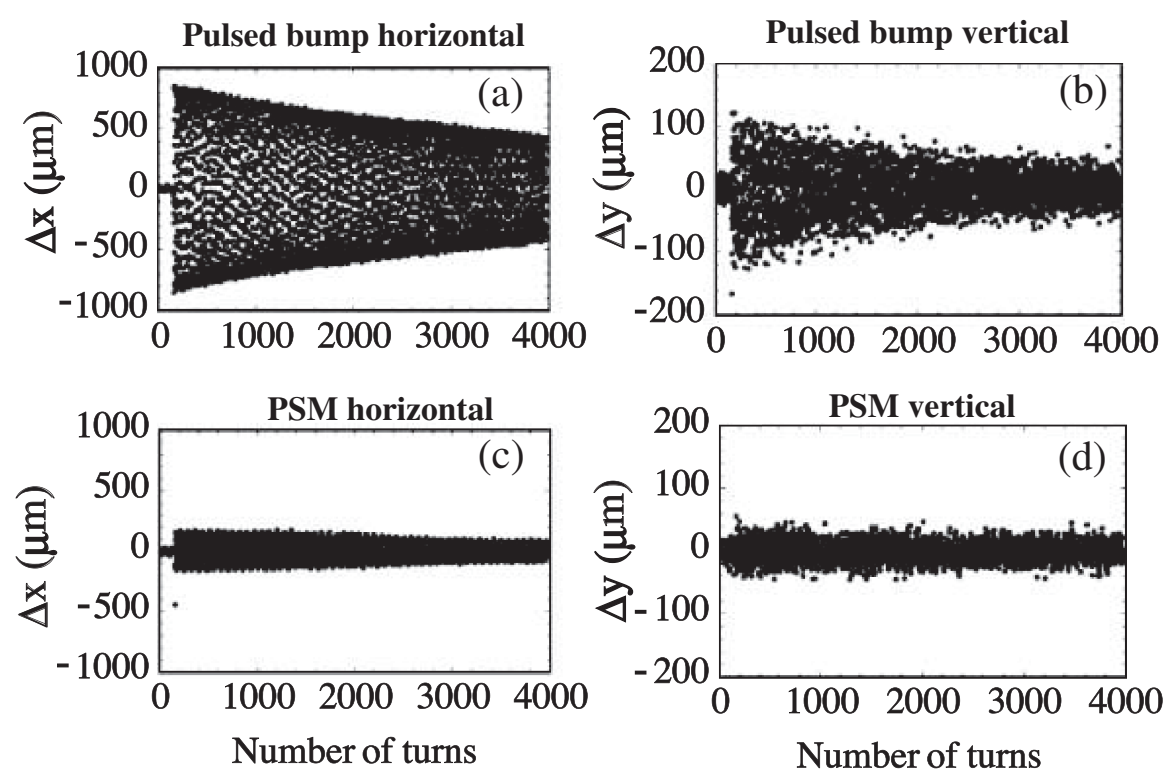

FIG. 20. Horizontal and vertical beam oscillations ( $\Delta x$ and $\Delta y)$ of the stored beam immediately after injection. The oscillations produced by the pulsed bump are shown in the upper two figures, (a) and (b), and those by the PSM are in the lower two figures, (c) and (d). 

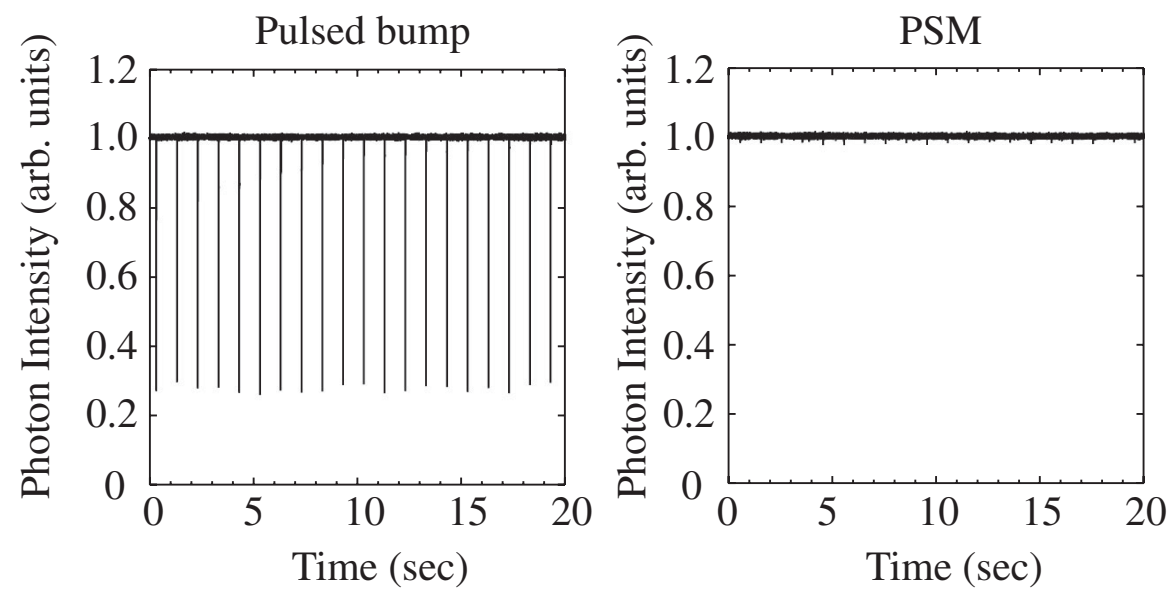

FIG. 21. Stability of photon intensity at BL-14A in the pulsed bump injection (left graph) and the PSM injection (right graph). The spike train appeared at exactly $1 \mathrm{~Hz}$ in both graphs, synchronized with the beam injection. The sampling rate of the signals was $10 \mathrm{kHz}$.

which exactly synchronized with the beam injection. For the pulsed bump injection, the maximum change of the photon intensity was very large, about $70 \%$. On the other hand, it became only $\sim 1 \%-2 \%$ for the PSM injection. This result shows that the PSM injection is very effective for top-up injection.

BL-5A and BL-17A have horizontal undulators. The photon intensity was measured at these undulator beam lines in the same run as the BL-14A experiment. In each beam line, a PIN photodiode was placed after focusing the source image, and a slit with a width of $50 \mu \mathrm{m}$ was used for BL-17A and $200 \mu \mathrm{m}$ for BL-5A. The sampling rate of the signal was $100 \mathrm{~Hz}$. The maximum change of the photon intensity during the pulsed bump injection was $80 \%$ at BL$17 \mathrm{~A}$ and $35 \%$ at BL-5A. On the other hand, during the PSM injection, the maximum change was $25 \%$ at BL-17A and was $7 \%$ at BL-5A. The differences of the maximum changes of the photon intensities mainly come from the size of the slit width. The PSM beam injection also improved the photon intensity stability at these beam lines compared with the pulsed bump injection.

\section{CONCLUSION}

We developed and installed a new beam injection system with a PSM at the PF ring and conducted beam injection experiments. We succeeded in injecting and storing the electron beam of up to $450 \mathrm{~mA}$. Top-up injection was also carried out without difficulty, and the stability of the stored beam current was less than $0.02 \%$ in peak to peak during two hours. Furthermore, we confirmed the greatest advantage of the PSM, i.e., the coherent dipole oscillations of the stored beam in both the horizontal and vertical directions are much smaller than those of the pulsed bump injection system. The measured maximum amplitude of the stored beam oscillation in the injection was much reduced by the
PSM injection in both horizontal and vertical directions. It was also shown by photon intensity measurement at three SR beam lines that the photon intensity stability could be much improved by the PSM injection. We successfully demonstrated the PSM injection and its advantages at the PF ring.

\section{ACKNOWLEDGMENTS}

The authors acknowledge the staff of the Light Source Division of the Photon Factory and the Synchrotron Radiation Laboratory of ISSP. Especially, we thank Dr. S. Kishimoto and Dr. Y. Yamada who provided experimental data of beam lines. We also thank Professor T. Kasuga and Professor A. Kakizaki. This work was supported by a Grant-in-Aid for Scientific Research (C) (KAKENHI 18540304).

[1] H. Ohkuma, in Proceedings of the 11th European Particle Accelerator Conference, Genoa, 2008 (EPS-AG, Genoa, Italy, 2008), p. 36.

[2] H. Tanaka, T. Ohshima, K. Soutome, and M. Takao, Nucl. Instrum. Methods Phys. Res., Sect. A 539, 547 (2005).

[3] Y. Kobayashi and K. Harada, in Proceedings of the 10th European Particle Accelerator Conference, Edinburgh, Scotland, 2006 (EPS-AG, Edinburgh, Scotland, 2006), p. 3526.

[4] H. Takaki, N. Nakamura, Y. Kobayashi, K. Harada, T. Honda, T. Miyajima, and S. Nagahashi, in Proceedings of the 2007 Particle Accelerator Conference, Albuquerque, New Mexico, 2007 (IEEE, Albuquerque, New Mexico, 2007), p. 231.

[5] H. Takaki, N. Nakamura, Y. Kobayashi, K. Harada, T. Miyajima, A. Ueda, S. Nagahashi, T. Honda, and T. Obina, in Proceedings of the 11th European Particle Accelerator Conference, Genoa, 2008 (Ref. [1]), p. 2204. 
[6] K. Harada, Y. Kobayashi, T. Miyajima, and S. Nagahashi, Phys. Rev. ST Accel. Beams 10, 123501 (2007).

[7] K. Hirata, CERN Report No. 88-04.

[8] POISSON/SUPERFISH, Los Alamos National Laboratory Report No. LA-UR-96-1834.
[9] A. Ueda, T. Ushiku, and T. Mitsuhashi, in Proceedings of the Particle Accelerator Conference, Chicago, IL, 2001 (IEEE, New York, 2001), p. 4050. 